\title{
A full scale propeller wash erosion test on heterogeneous cohesive material
}

\author{
Y. Karelle \\ Dredging International nv, Zwijndrecht, Belgium \\ P. Sharpe \\ Bechtel Oil, Gas \& Chemicals Inc, Perth, Australia
}

GM. Pizzo

Chevron Australia Pty Ltd, Perth, Australia

P. Haerens

International Marine and Dredging Consultants nv, Antwerp, Belgium

V. Albanese

HR Wallingford Ltd, Wallingford, UK

\begin{abstract}
As part of a life-cycle cost analysis for the construction of a harbour basin, a full scale erosion test on a dredged slope was undertaken using a test vessel. The objective of the test was to estimate the erosion rate of the in-situ material in order to predict the final profile of the dredged slope at the end of the design life following exposure to propeller wash generated by various manoeuvring vessels. Based on theoretical formulae, a test protocol of percentages of the installed engine power to be applied was defined, aiming to generate a steady propeller flow on the slope as a function of the tide. A pre-installed anchoring point ensured the test vessel maintained a steady position during the test and the pre-defined percentages of the installed engine power were applied as a function of the tidal levels measured during the test. Several interim bathymetric surveys were undertaken in order to monitor and record the progress of the erosion over the tested slope. Orientation and location of the vessel were monitored by satellite positioning, tides were recorded by installed gauges and actual flow velocities logged by a current meter. A time stamped geo-database was developed to model the theoretical distribution of the velocities at any moment of the test. Through the analysis of the tests results, a curve defining the erosion rate as a function of the velocity at the slope was derived from the model and was verified by comparing generated cross-sections against actual surveys. Results were then extrapolated to represent the situation at the end of the design life of the harbour in order to assess whether protection of the slopes was required to preserve the integrity of the marine structures adjacent to the harbour basin. Results were also used to define an appropriate monitoring program for the dredged slopes which had potential for scour generated by propeller wash.
\end{abstract}

\section{INTRODUCTION}

Traditionally, a scour protection system has to be foreseen within harbour facilities in order to prevent potential negative effect of erosion caused by propeller wash action. In fact, the extent of the developing scour over the design life of the facilities could cause geotechnical instability and even failure of a structure exposed to propeller wash if not protected (PIANC 2015). As part of a life-cycle cost analysis for the construction of a harbour basin, the development of the scour holes caused by propeller wash on the basin dredged slopes was expected to be limited; for this reason it was a possibility that scour protection would not be required. In order to support such a decision it was necessary to accurately define the expected navigation manoeuvres within the harbour; therefore, information regarding the berthing vessels characteristics, manoeuvring path, duration and frequency were identified.

A geotechnical assessment defining the requirement for maintaining the integrity of the structures 


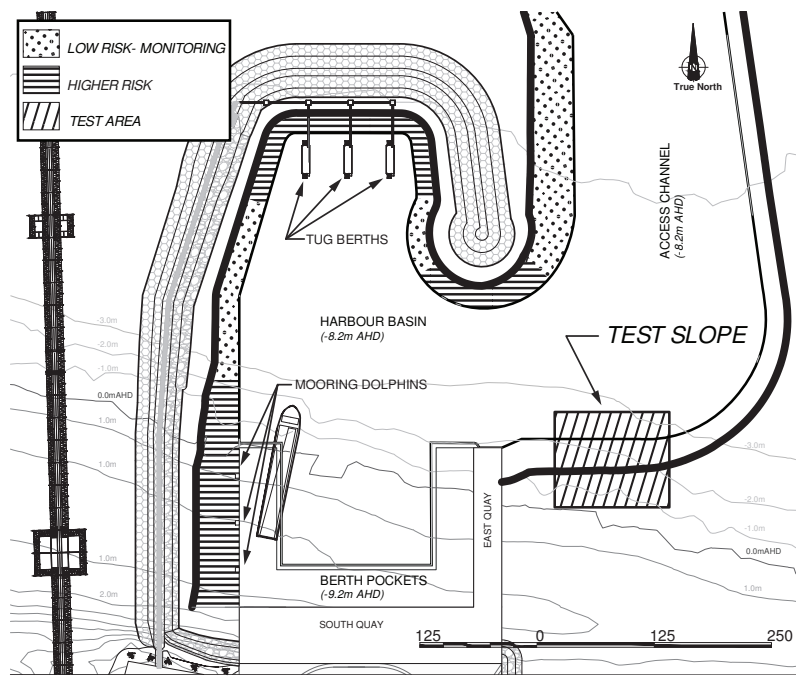

Figure 1: Harbour basin plan layout. Estimation of scour erosion risk (from "low-risk" to "higher risk") by navigation manoeuvres simulation and represented by different hatches. The test slope is also indicated.

adjacent to the dredged slopes was also completed in order to set the boundary condition of what can be considered acceptable erosion.

Lastly, information on the erodibility behaviour of the subsoil subject to propeller wash action was essential to predict the evolution of the dredged slope over time. The navigation and geotechnical information were defined with confidence at the early stage of the design process. On the contrary the erodibility has required more effort, due to limited available literature and uncertainties inherent to the heterogeneity and cohesive property of the in-situ material; for those reasons it was proposed to undertake a full-scale erosion test with a test vessel on a nearby slope of similar geometry and soil characteristics (location of the test slope is shown on Figure 1).

The objective of the test was set as estimating the erosion rate of the in-situ material in order to predict the dredged slope profile at the end of the design life of the harbour basin exposed to propeller wash generated by various manoeuvring vessels.

\section{SITE SPECIFICS}

\subsection{Harbour basin and test location}

The harbour basin is dredged down to a level of -7.0 $\mathrm{mCD}$ and is delimited by dredged slopes of $1 \mathrm{~V} / 4 \mathrm{H}$ gradient. In the vicinity of the dredged slopes, a breakwater is built. Based on the results of a manoeuvring simulation, the dredged slopes presenting risk in term of propeller wash were defined (higherrisk slopes, see Figure 1, in opposition to "low-risk" slopes). The test slope presented a similar dredge slope gradient and stratigraphy to that of the higherrisk slopes and was not adjacent to any marine structures.

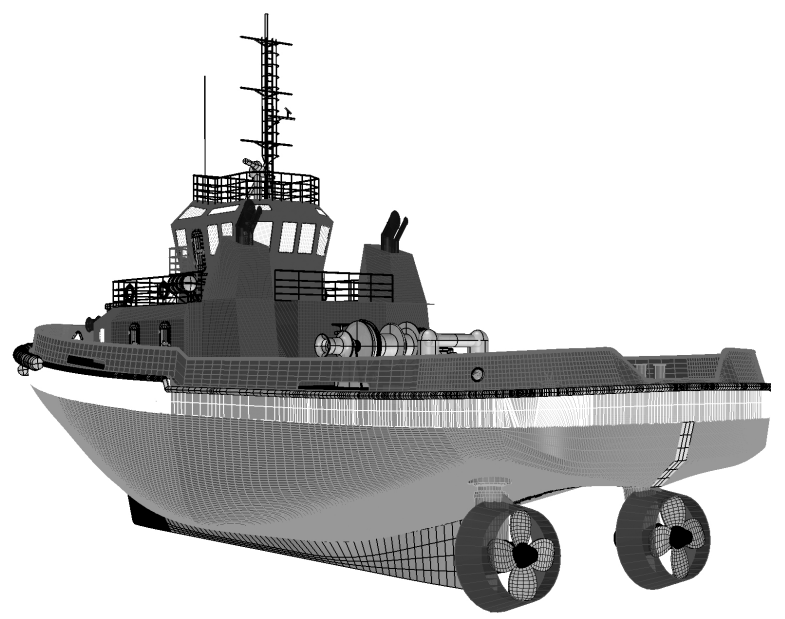

Figure 2: Tug boat with twin ducted propellers, used as test vessel.

Table 1: Design and Test vessels characteristics.

\begin{tabular}{lll} 
Characteristics & Design vessel & Test vessel \\
\hline Engine Power $(\mathrm{kW})$ & 4500 & 3730 \\
Propeller Diameter $(\mathrm{m})$ & 4 & 2.4 \\
Draft $(\mathrm{m})$ & 9 & 4.7 \\
Ducted propellers & Yes & Yes \\
Number of propeller & 2 & 2 \\
\hline
\end{tabular}

\subsection{Design vessel}

Based on the results of a navigation simulation within the harbour basin, a design vessel (with characteristics reported in Table 1) was defined as being the vessel that is expected to cause the largest propeller wash when normally manoeuvring in proximity of the higher-risk slopes.

The number of expected manoeuvres over the entire design life of the harbour was also estimated. The duration of the propeller wash attack, the positions of the design vessel and applied power during manoeuvring in proximity of each dredged slope were evaluated from the navigation simulation.

\subsection{Test Vessel}

A test vessel was chosen for the full-scale test in order to create similar velocities to the design vessel at the seabed and dredged slope. A large tugboat with twin ducted propellers was selected (characteristics are also reported in Table 1). A model of the test vessel is shown in Figure 2.

\subsection{Stratigraphy}

From boreholes executed in the vicinity of the dredged slopes and from the results of the laboratory tests, the in-situ material was classified as being heterogeneous and mainly consisting of two types of material. Type 1 material was classified as clayey sand to silty sand with low plasticity, with a $d_{50}$ varying from 150 to $190 \mu \mathrm{m}$; a fines content between $9 \%$ and $35 \%$ and a gravel content between $0 \%$ and $13 \%$. Particle size distribution (PSD) results of the Type 1 material are presented in Figure 3. 


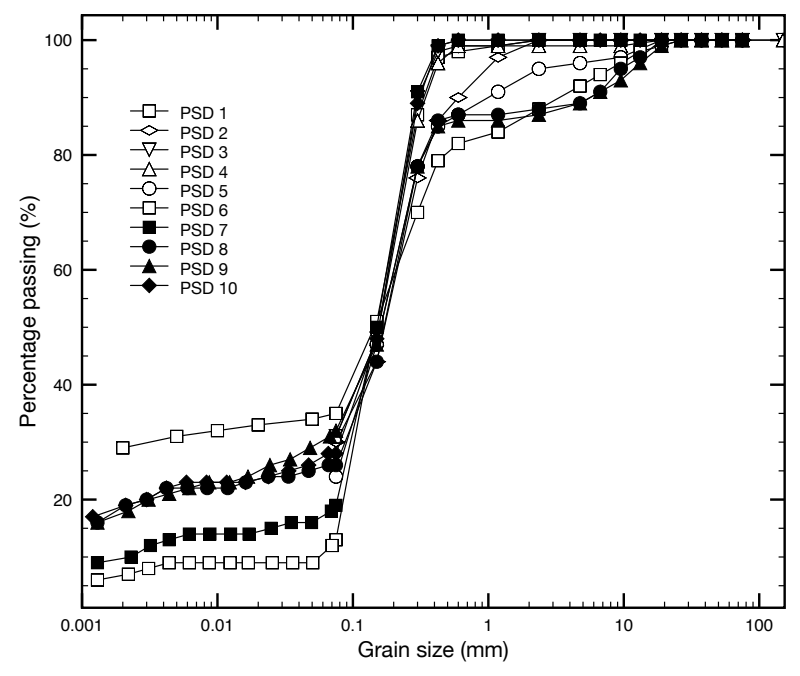

Figure 3: Summary of PSD results for Type 1 material found on test slope.

Type 2 material was found in pockets located within the Type 1 material of fine to medium gravel with clay lenses and calcareous sandstone recovered as gravel / cobbles. Due to the presence of Type 2 material found in the form of pockets, it was not possible to assess the location of Type 2 material along the Test slope alignment with a satisfactory accuracy before proceeding with the full-scale test.

\section{TEST DESCRIPTION}

The full-scale test was staged as follows:

1. Definition of theoretical efflux velocity from propellers, of the velocity distribution in the jet and of the velocity at the seabed and at the dredged slope due to vessel action;

2. Definition of the test setup, protocol and instrumentation; and

3. Execution of the full-scale test with appropriate data collection.

\subsection{Theoretical propeller wash flow field}

The resulting velocities on the seabed and dredged slopes were calculated based on the Dutch Approach. The Dutch Approach is itself based on the actuator disc theory assuming that the propeller jet can be considered as a submerged free jet, discharging out of an orifice into an infinite fluid (Albertson et al. 1950). It results in the calculation of the efflux velocity $V_{0}$ :

$V_{0}=C \sqrt[3]{\frac{f P_{B}}{\rho D_{P}^{2}}}$

where $C$ is the propeller clearance in metres; $f$ is the ratio engine power in $\mathrm{kW} ; P$ is the engine power in $\mathrm{kW} ; \rho$ is the water density in tonnes per cubic metre; and $D_{P}$ is the propeller diameter in metres.

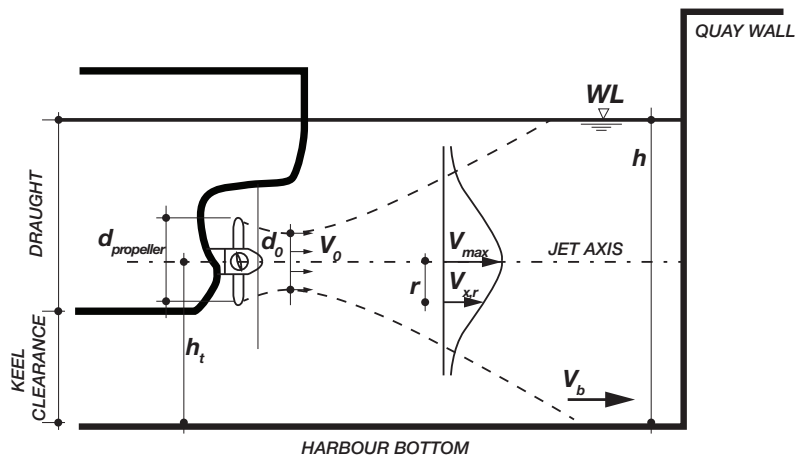

Figure 4: Definition for jet of the main propulsion system (PIANC 2015).

Thereafter a 3D propeller wash flow field $V_{x, r}$ can be built by first calculating the decreasing velocity along the propeller axis $V_{a x i s}$, and subsequently applying a radial Gaussian distribution to calculate $V_{x, r}$ (Verheij 1983):

$$
\begin{aligned}
& V_{a x i s}=C V_{0}\left(\frac{D_{P}}{x}\right) \\
& V_{x, r}=e^{-15.4 \frac{r^{2}}{x^{2}}} V_{a x i s}
\end{aligned}
$$

where $C$ is equal to 2.8 for a ducted propeller and 1.95 for a non- ducted propeller; $x$ is the axial distance in metres; and $r$ is the radial distance in metres.

Due to the presence of two propellers (for both the design and test vessels), the resulting velocity in a flow field caused by two propellers was obtained by linear superimposition of the flow field caused by each propeller separately (BAUW 2005).

\subsection{Test Protocol}

A test protocol was defined based on using the test vessel in order to achieve the highest expected velocity caused by the design vessel on the seabed and on the higher-risk slope. A total test duration of approximately 20 hours (representing several years of harbour operations) was considered to provide sufficient information to calibrate an erosion rate curve. During the test, the tide was expected to vary and, therefore, adjusted powers to be applied in function of the actual water level were defined with the intent of generating a close to steady propeller flow on the slopes and ensuring the target velocity was attained as consistently as feasible.

\subsubsection{Test setup}

A seven tonnes delta flipper anchor was deployed at the top of the test slope and connected to the towing wire of the test vessel (tug boat). The length of the towing wire was such to allow the test vessel to be located $20 \mathrm{~m}$ away from the toe of the test slope to achieve the desired velocities. A schematic description of the test setup is depicted in Figure 5. 


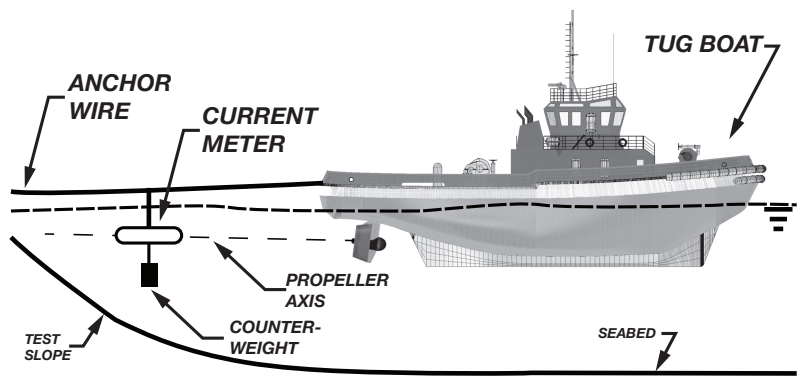

Figure 5: Test vessel is located $20 \mathrm{~m}$ away from the test slope during the full-scale test. The vessel is maintained in a steady position with a seven tonnes delta flipper anchor located at the top of the slope. The current meter is located as close as feasible to the axis of the propellers.

\subsubsection{Instrumentation}

A current meter (type Valeport Model 308) was installed on the towing wire to provide qualitative assessment of the velocities near the propellers. The current meter was suspended so that it could direct towards the current freely by using a swivel, was able to move 25 degrees for pitch and roll and was vertically maintained by a counter-weight. It was located as close as feasible to the axis of the propellers.

\subsection{Full scale test}

The full-scale test was carried out in accordance with the protocol described in Section 3.2. The power applied was adjusted with the actual tide reading and the start and stop times of adjustments were recorded. In addition, the following information was logged at an interval of five seconds by a digital satellite positioning system:

- Northing and Easting of the port and starboard sides propellers, in metres;

- Heading of the vessel, in degrees; and

- Actual tide reading, in metres chart datum.

An in-survey prior to the full-scale test and a final out-survey post-test were carried out. In addition, a total of thirty interim surveys were conducted in order to monitor the evolution of the erosion caused by propeller wash action every 45 minutes of cumulated testing time on average.

\section{MODEL SETUP}

\subsection{Data collection}

Upon completion of the full-scale test, a model consisting of a time stamped geo-database linked with a GIS software was set up in the first stage to collect the metadata for all the logged information. In the second stage, the model was used to assess the theoretical erosion impact of any position of the vessels at any points on the seabed and on the test slope, during the full-scale erosion test. A grid $0.5 \mathrm{~m}$ by $0.5 \mathrm{~m}$

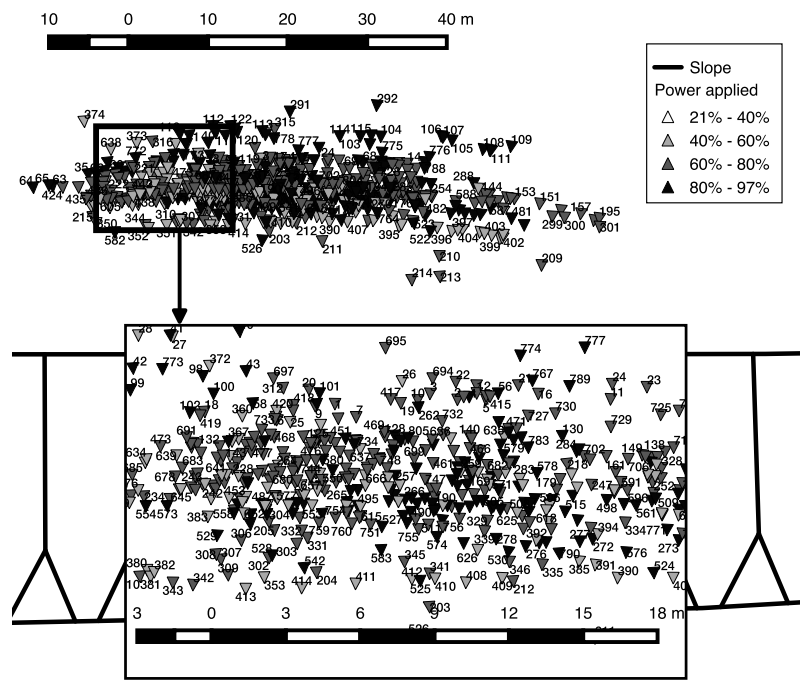

Figure 6: Representation of the 806 vessel positions during the full-scale test. The arrows represent her position over time, the arrows directions represent the orientations (headings) of her propellers and the colours are graded in function of the different power applied. A box is included to provide more details in the zone of higher positions density, i.e. where the test vessel was predominantly positioned during the full-scale test.

was defined based on the consideration for the accuracy vs. calculation time criteria. An area of approximately $50,000 \mathrm{~m}^{2}$ was covered by the model. In addition, the vessel positions were regrouped and averaged over a period of similar water level using 0.1 $\mathrm{m}$ bins. A total of 806 vessel positions, orientations and associated propeller power were considered. An identification tag (ID) was assigned for each position in chronological order. Importantly, it allowed identifying the time-line of the different positions of the vessel against the interim surveys.

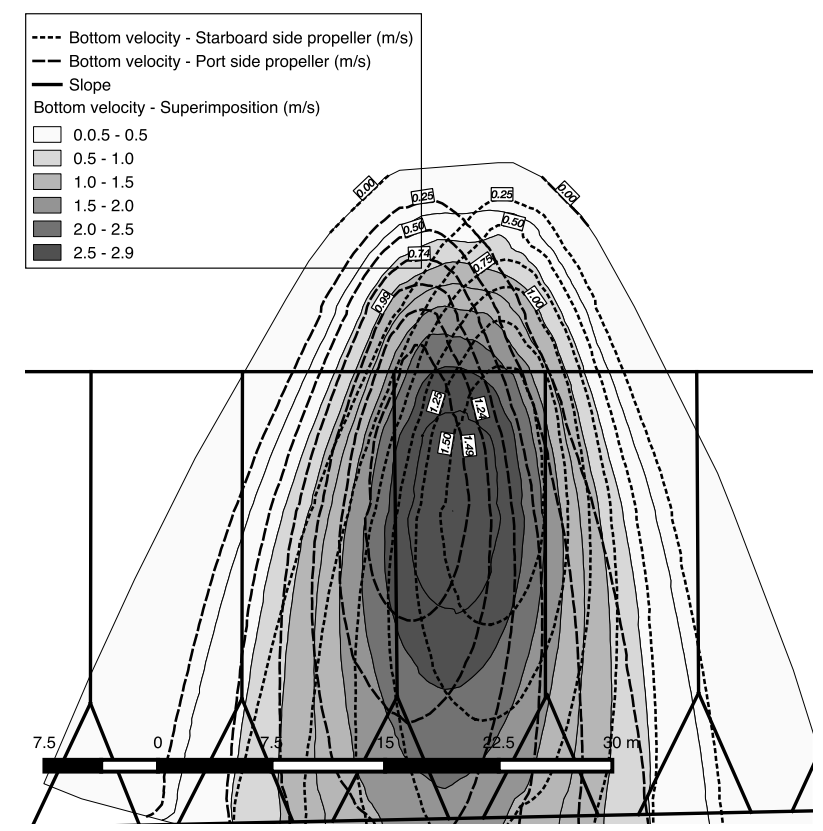

Figure 7: Representation of the theoretical flow field (caused by the twin propellers for one vessel position) calculated by the $3 \mathrm{D}$ model at the bottom (seabed or slope). 


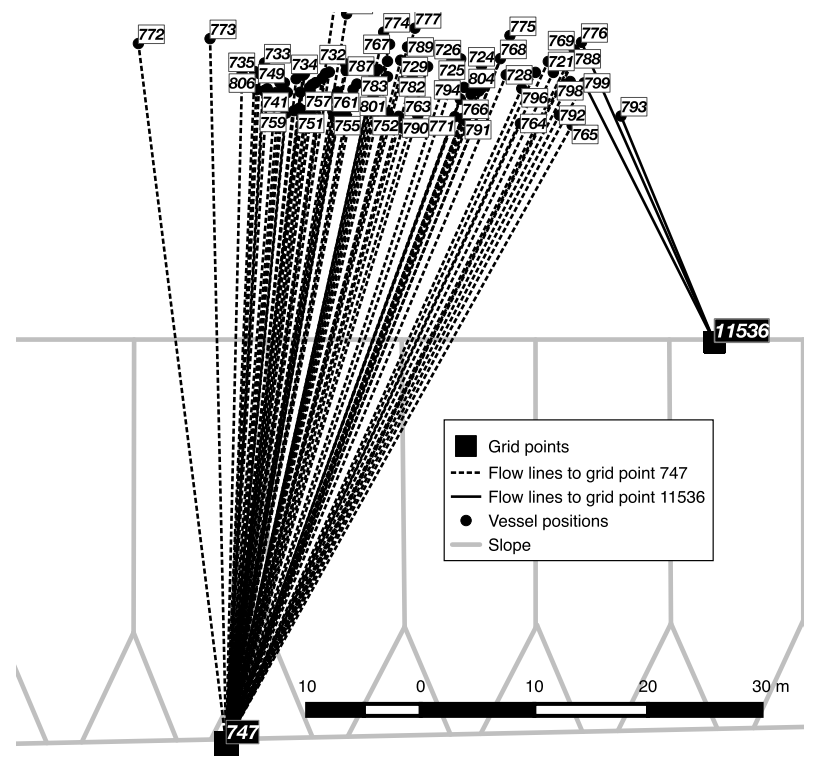

Figure 8: Representation of vessel positions that theoretically impact two typical grid points with velocities $>0.05 \mathrm{~m} / \mathrm{s}$. One point is greatly exposed (grid point 747, located at the top of the slope) and one is marginally impacted by the full-scale test (grid point 11536 located at the eastern part of toe of the test slope). For the sake of readability, only few vessel positions are depicted.

\subsection{Implementation of the velocities}

The theoretical velocities evaluated from equations (1), (2) and (3) as described in Section 3.1 were calculated for each tug position and recorded in the database. It allowed the evaluation of the theoretical velocity distribution on the seabed and dredged slope for the different test vessel position (as defined in Section 4.1) during the full-scale test. The orientation of the vessel was also taken into account. An example of velocity distribution for one typical position is given in Figure 7. It can be seen that in this particular example, the vessel had an orientation of approximately five degrees from the North-South axis.

Velocities lower than $0.05 \mathrm{~m} / \mathrm{s}$ were assumed to be below the erosion trigger of the in-situ material and were, therefore, discarded. The velocity distribution for every point of the grid was calculated for every position of the vessel.

A total of $10.9 \times 10^{6}$ velocities were calculated and recorded in the database for future analysis, and were calculated to be within a range between 0.05 and 3.26 $\mathrm{m} / \mathrm{s}$.

In Figure 8, an example is given for two typical grid points (one being greatly exposed, the other one lightly exposed to the different position of the test vessel). The lines connect the 2 two grid points with the different positions of the vessel having theoretically induced velocities greater or equal to $0.05 \mathrm{~m} / \mathrm{s}$ at these locations.

A qualitative comparison between the logged data from the current meter and the flow velocities calculated by the $3 \mathrm{D}$ model was carried out and is represented in Figure 9. From the test vessel orientation $\log$, it was assessed that the vessel did not maintain a perfect alignment with the anchor position, result-

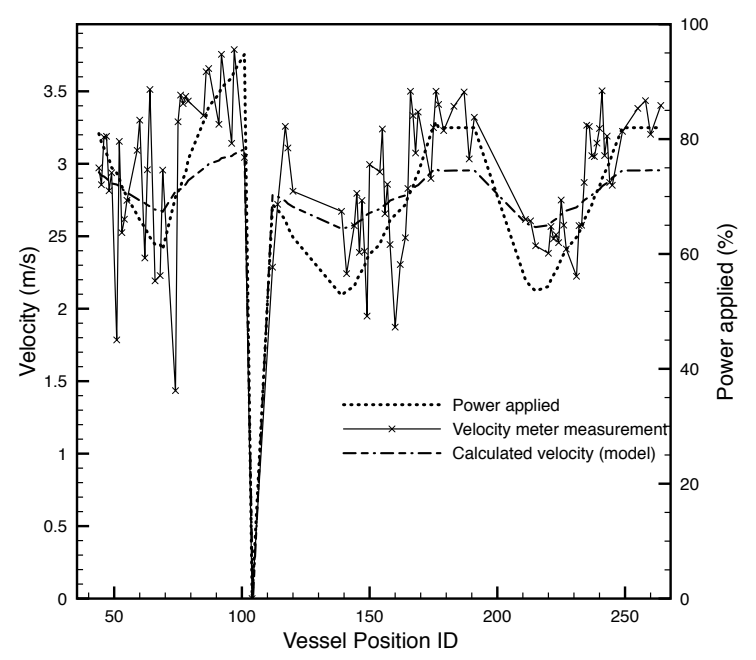

Figure 9: Comparison of the velocities logged by the current meter and the theoretical velocities calculated by the 3D model at the location of current meter, assuming the current meter is in the alignment of the propeller axis. The logged applied power is also represented.

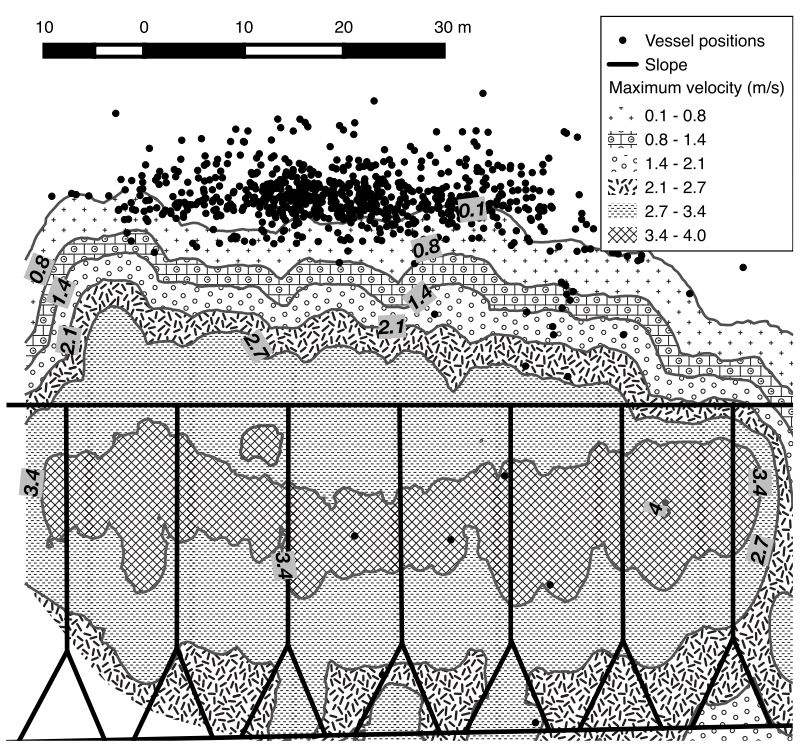

Figure 10: Maximum velocities having affected each grid points over the entire full-scale test duration. It is noted this plot does not indicate the duration during which the maximum velocities were achieved.

ing in some misalignment of the current meter with the test vessel propeller horizontal axis (average orientation of 0.34 degree with a standard deviation a 4.3 degree). In addition, based on the observation of oscillating flow velocities recorded in the current meter $\log$, it was deduced that the current meter had been subject to a pendulum movement during the test. Given the above, it was concluded that the comparison provided, on average, a satisfactory qualitative validation of the model flow velocity distribution and that the pattern of velocities as recorded by the current meter was in line with the pattern of recorded adjustments of the power applied. 


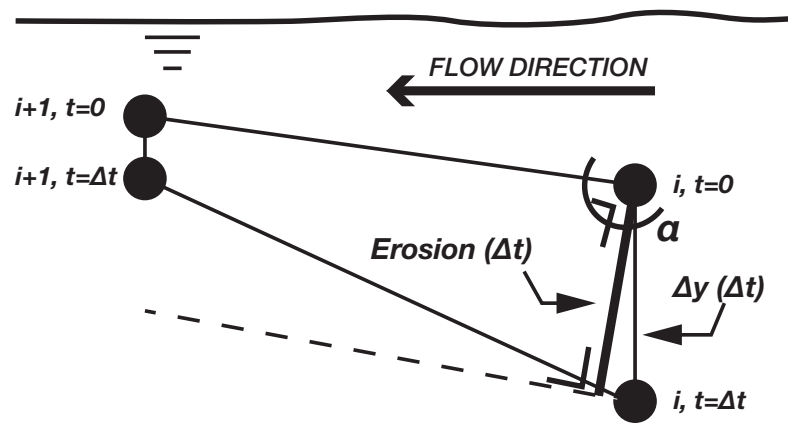

Figure 11: Erosion is calculated based on the geometry of the terrain, with a slope evaluated from upstream to downstream.

\section{ANALYSIS}

\subsection{Maximum achieved velocities}

From an analysis of the theoretical velocity distribution induced by all of the 806 positions of the test vessel, it was concluded that the pre-defined protocol allowed the maximum target velocity to be achieved on 9,000 grid points (i.e. $70 \%$ of the tested surface). The distribution of the maximum achieved velocity over the test slope is given in Figure 10.

\subsection{Definition of the erosion model}

In order to account for the evolution of the terrain, the erosion $E_{i}$ was implemented in the model as depicted in Figure 11, instead of using the vertical level difference $\Delta y_{i}$.

The erosion of a grid point located at a chainage $i$ during a period $\Delta t$ is calculated as a function of the slope of the terrain between the grid point $i$ and the downstream grid point $i+1$. Therefore, the erosion $\left(E_{i}\right)$ for a grid point $i$ during a period $T$ can be expressed as follow:

$E_{i}=\sum_{t=0}^{T}\left(\Delta y_{i}\right)_{t} \sin \left(\alpha_{(i, i+1)}\right)_{t}$

\subsection{Range of velocities between two surveys}

Erosion $E_{i}$ due to propeller wash (in metres, as defined in Section 5.2) occurring at a certain grid point can also be obtained directly by comparing two surveys covering the considered grid point. This assumption is justified since the metocean conditions were calm during the test. Thus this erosion was assumed to have been caused by flow velocities induced by the test vessel, during the time window delimited by the two surveys and not by other actions (such as tide or storm current). If we consider now the fact that the erosion rate is defined as being the erosion by unit of time as a function of the flow velocities, we can conclude that a calculated erosion $E_{i}$ can serve as a relevant input for the calibration of an erosion rate curve if the flow velocity impacting the grid point between a pair of surveys is known.

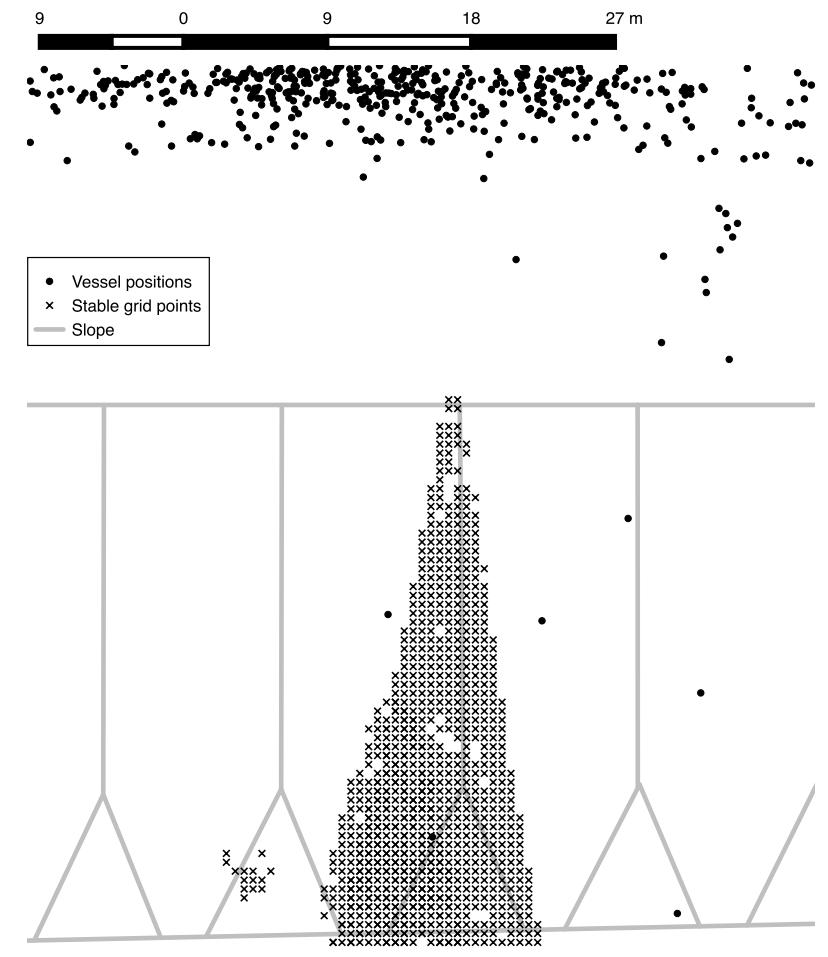

Figure 12: Location of the grid points providing relevant data (i.e. grid points having been impacted by velocities in the same range of magnitude, criteria being average velocity/standard deviation ratio $<20 \%$ during a period delimited by the execution of two consecutive surveys) for the purpose of calibration.

Unfortunately despite the fact that surveys had been carried on regular basis, it was identified that each grid point had been subject to velocities resulting from an average of seven to eight adjustments of power between two surveys in order to account for the water level changes. In addition, the vessel did not maintain a constant orientation.

Therefore, it was concluded that each grid point had been potentially subject to a range of varying velocities between surveys. Due to the non-linear behaviour of the erosion as a function of the flow velocity, it was concluded that using the average velocity to determine an erosion rate curve was not applicable and a strategy had to be defined to overcome those issues.

\subsection{Definition of a first erosion rate curve}

\subsubsection{Relevant data}

Due to the difficulties reported in Section 5.3, a selection of only relevant data had to be made in order to calibrate a first curve. Erosion of grid points having been impacted by velocities in the same range of magnitude (average velocity/standard deviation ratio $<20 \%$ ) during a period between two consecutive surveys were considered as relevant data. Figure 12 shows the locations of grids points providing relevant data for the purpose of calibration.

It is noted that grid points depicted in Figure 12 could have met the above criteria for a different pair of surveys, thus providing more than one set of relevant data. The erosion $E_{i}(\mathrm{~m})$ of the selected relevant grid 
Table 2: Value of regression coefficients $\alpha$ and $\beta$ deduced from (Briaud 2008) for sand and clay material.

\begin{tabular}{lll} 
Material & $\alpha$ & $\beta$ \\
\hline Sand & 11.78 & 5.63 \\
Clay & 1.25 & 2.91 \\
\hline
\end{tabular}

points were then converted to an erosion rate $\left(R_{i}(v)\right)$ by dividing their values with the cumulative testing time occurring during the time window delimited by the execution of a pair of consecutive surveys.

$R_{i}(v)=\left(\sum_{t=0}^{T} \frac{\left(\Delta y_{i}\right)_{t} \sin \left(\alpha_{(i, i+1)}\right)_{t}}{t}\right)$

Those erosion rates $R_{i}(v)$ expressed in $\mathrm{mm} / \mathrm{h}$ were then plotted as a function of their associated average near-bed flow flow velocities (expressed in $\mathrm{m} / \mathrm{s}$ ) as defined by equation 5 and reported in Figure 13.

\subsubsection{Analytic formulation}

The described method in Section 5.4.1 provides a way to plot erosion rate cloud values $R_{i}(v)$ deduced from experimental data of the full-scale test. Thereafter an analytic formulation can also be defined. Briaud (2008) provides erosion rate $R(v)$ curves for sand and clay material which were obtained from lab testing. An analytic regression of those curves was estimated and was also adopted as the standard form of the erosion rate $R(v)(\mathrm{mm} / \mathrm{hr})$ equation for the in-situ material:

$R(v)=\alpha v^{\beta}$

where $\alpha$ and $\beta$ are the coefficient to be calibrated, depending on the type and heterogeneity of the soil and $v$ the velocity in $\mathrm{mm} / \mathrm{s}$.

Based on the plot of the relevant experimental data as defined in equation 5 and plot in Figure 13, coefficients $\alpha=18$ and $\beta=3.7$ were considered as a first assessment of an erosion rate $R(v)$ curve for the insitu material. This assumption was considered conservative since it provided higher erosion rates than the actual computed value by the model as can be seen in Figure 13.

\subsection{Validation and optimisation of the curve}

In order to validate the first erosion rate curve, a time window had to be selected carefully. With reference to Section 5 , a time window is mainly defined by a pair of surveys (which can be either consecutive or non-consecutive). A pair of surveys was, therefore, selected based on vessel positions (as steady as possible) and orientation (as constant as possible) adopted by the test vessel during the time window delimited by the two surveys. Moreover, the two surveys had to be sufficiently spaced in time, in order to limit the effect of erosion/deposition phenomenon. Therefore,

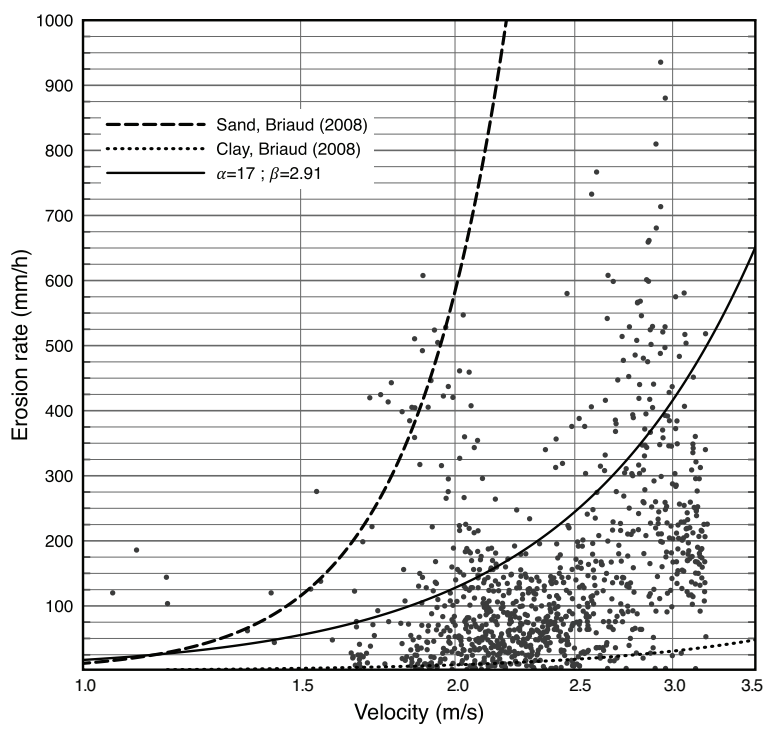

Figure 13: Plot of the first erosion rate curve $R(v)(\alpha=18, \beta=$ 3.7). The erosion rate curve for sand material and clay material are derived from (Briaud 2008). The cloud of dots represents the relevant data $R_{i}(v)$ (Section 5.4.1).

the pair of surveys were not required to be consecutive (in opposition to the approach taken for calibration purpose as described in Sections 5.4.1 and 5.4.2), i.e. interim surveys could have occurred in between the selected surveys. It was also verified that the relevant data used for the calibration exercise (described in Section 5.4.1) were not due to flow velocities that occurred in the time window used for validation.

In order to validate the erosion rate curve, an additional erosion module was added to the model. Starting from the bathymetry of the first survey of the pair (i.e. considered as the in-survey), the bathymetry of each grid points was lowered by the cumulative erosion $\sum E_{i}$ (erosion $E_{i}$ being calculated with the defined first erosion rate curve) due to the flow velocities induced by the different positions of the vessel. Thereafter, the updated bathymetry was compared to the second survey of the pair (i.e. considered as outsurvey) on generated cross profiles every $50 \mathrm{~cm}$ (examples are given in Figure 14).

It was concluded that the first erosion rate $R_{i}(v)$ curve $(\alpha=18$ and $\beta=3.7)$ provided an over estimation of the erosion. Also it was observed that a layer of material with very low erodibility property was present over a length of approximately $40 \mathrm{~m}$ and with depth varying between $-3.2 \mathrm{~m}$ and $-7.0 \mathrm{~m}$. This was based on negligible differences between the in and out surveys at those locations, despite the fact they had been exposed to the same range of velocities than the more eroded location. This low erodability property was associated with Type 2 material (see Section 2.4).

Therefore, it was proposed to split the validation time window in two groups of approximately equivalent testing time and to use the first group to recalibrate the erosion rate curve for Type 1 material by adjusting the coefficient $\alpha$ and $\beta$ to obtain an estimated eroded profile as close as possible to the out- 

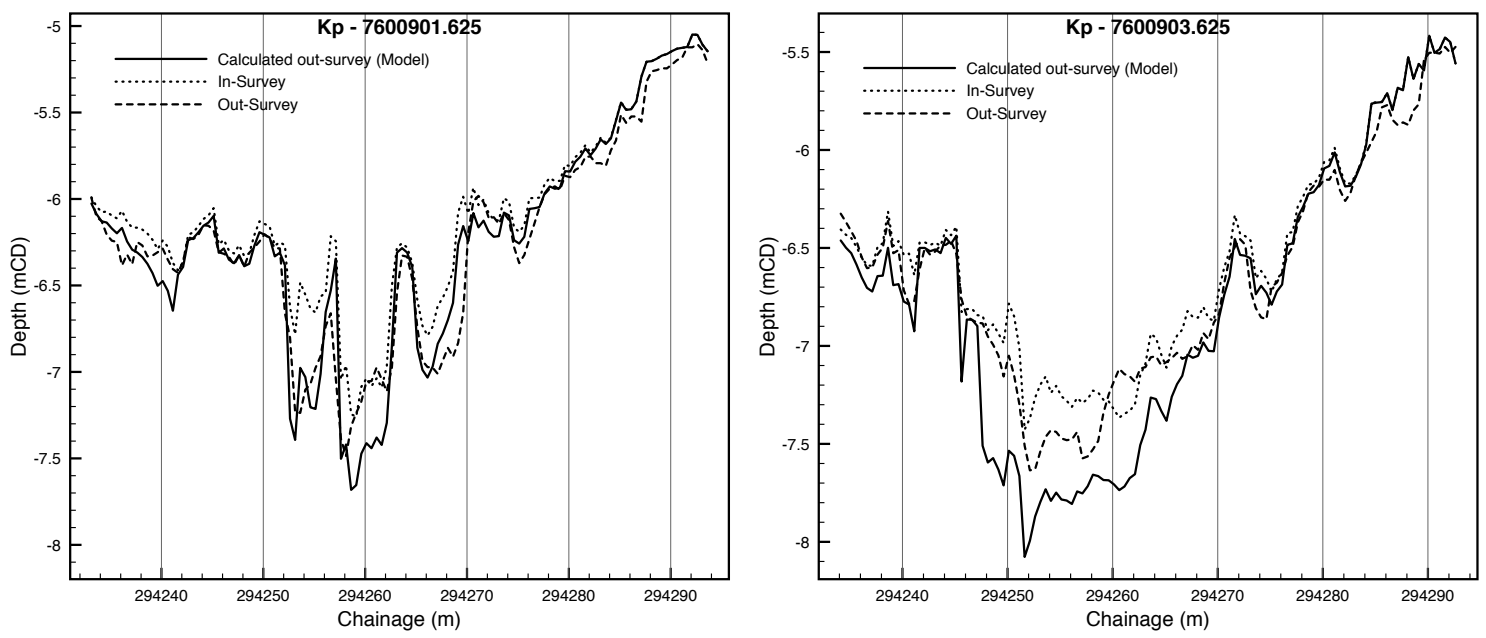

Figure 14: Generated cross profiles by the 3D model, between a pair of in and out surveys. The erosion $E_{i}$ is evaluated using the optimized erosion rate $R(v)$ curve $(\alpha=17$ and $\beta=2.61)$ defined in Section 5.5, maintaining a certain conservatism being required for the purpose of the study. It can be observed on the out-survey curve that the local terrain depression existing in the in-survey are being amplified and slightly shifted in the downstream direction. This is also obtained in the calculated out-survey by the 3D model. This is attributed to the adopted methodology to evaluate the erosion $E_{i}$ as described in 5.2.

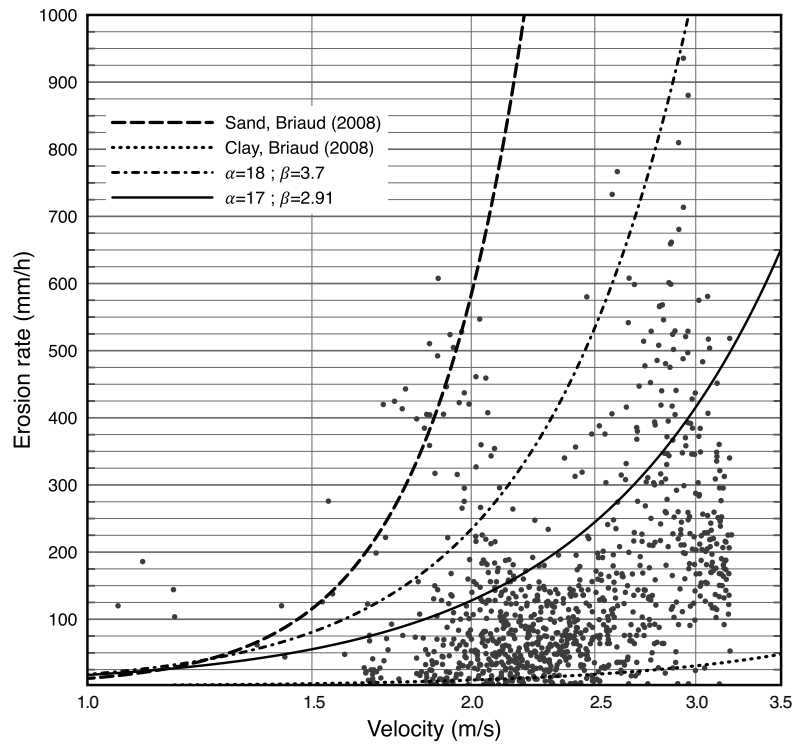

Figure 15: Plot of the best-fitting erosion rate curve $R(v)$ of Type 1 material $(\alpha=17, \beta=2.91)$. The erosion rate curve for sand material and clay material are derived from (Briaud 2008). The cloud of dots represents the relevant data $R_{i}(v)$ (Section 5.4.1).

survey (with sufficient conservatism, given the purpose of the study). The curve for clay as described in (Briaud 2008) was applied for the Type 2 material in order to model the observed low erodibility behaviour of that material. Finally, it was proposed to use the independent second group of tests to validate the curves for Type 1 and Type 2 materials.

The best-fitting curve for the material of Type 1 material was found for coefficients $(\alpha=17, \beta=2.91)$ and is depicted in Figure 15. It presents erosion rates in between those defined by Briaud (2008) for the sand and clay.

It was also concluded that for the range of tested velocities the Type 2 material was less erodible than the clay material defined in (Briaud 2008).
Table 3: Value of regression coefficients $\alpha$ and $\beta$ deduced from (Briaud 2008) for sand and clay material and best fitting curve for in-situ material deduced from full-scale test.

\begin{tabular}{lll} 
for in-situ material deduced from full-scal & $\alpha$ & $\beta$ \\
\hline Material & 11.78 & 5.63 \\
Sand (Briaud 2008) & 1.25 & 2.91 \\
Clay (Briaud 2008) & 17 & 2.91 \\
Best fitting curve & 17 & \\
\hline
\end{tabular}

\section{APPLICATION OF THE EROSION CURVE}

Once the calibration and validation of the erosion rate curve was completed, it was implemented in an erosion model aiming to determine the expected extent of the erosion at the higher-risk dredged slopes of the harbour basin, in the vicinity of the breakwater. The erosion model determined the expected eroded profile of the dredged slopes based on an estimation of the design vessel track, frequency and duration of manoeuvres and erosion rates defined by the calibrated curve.

It was assessed that the expected eroded profile was not foreseen to affect the geotechnical stability of the breakwater located in the vicinity and it was concluded that no scour protection was required since it did not impede on the critical part of the slope required for the stability of the breakwater. In addition, based on the ability to determine the theoretical chronological evolution of the dredged slope erosion, a monitoring program was set-up.

\section{SUMMARY}

As part of a life-cycle cost analysis for the construction of a harbour basin, a full scale erosion test on a dredged slope was undertaken using a representative vessel. The objective of the test was to estimate the erosion rate of the in-situ material in order to predict the final profile of the dredged slope at the end of the design life following exposure to propeller wash generated by various manoeuvring vessels. A test proto- 
col was developed in order to achieve the theoretical maximum velocity caused by a design vessel with a substitute test vessel of smaller size. Parameters were recorded during the execution of the test and were collected in a time stamped geo-database. Several interim bathymetric surveys were undertaken in order to monitor and record the progress of the erosion over the tested slope. A distribution of flow velocities were then calculated based on the Dutch approach formula for the different positions of the vessel during the test. It was assessed that the negative effect of the inherent execution difficulties (orientation of the vessel, tides, etc.) could be limited if the collected data is sufficient in quantity, clearly chronologically linked in a database and interim surveys are undertaken at an appropriate high-frequency. The impact of such difficulties could, therefore, be identified and discarded if necessary. The chosen analytical form of the erosion rate $E(v)$ curve of the in-situ material deduced from the experimental data collected during the fullscale test was similar to the analytical regression deduced from (Briaud 2008), who defined an erosion rate curve for sand (high erodibility) and clay material (low erodiblity):

$R(v)=\alpha v^{\beta}$

where $\alpha$ and $\beta$ are the coefficients depending on the type and heterogeneity of the soil and $v$ the velocity in $\mathrm{mm} / \mathrm{s}$.

A calibration dataset and an independent validation dataset were selected in order to define the best-fitting erosion rate curve. This was verified by generating cross-profiles, and comparing the in survey, out suvey and the expected erosion profile calculated from the defined erosion rate $R(v)$ curve.

This method allowed an erosion rate $R(v)$ curve to be defined that intrinsically included the in-situ behaviour of the materials, which would have been difficult to reproduce in laboratory due to the heterogeneity and cohesion of the subsoil generally encountered. In addition, the adopted methodology allowed identifying with accuracy the location of a pocket of material of low erodability within the predominant in-situ material constituting the test slope.

The best-fitting erosion rate $R(v)$ curve of the predominant material (Type 1 material, clayey sand to silty sand with low plasticity) was found to be in between the erosion rate $R(v)$ curves of the sand and clay material as defined in (Briaud 2008).

The pocket of low erodability material was associated with Type 2 material (fine to medium gravel with clay lenses and calcareous sandstone recovered as gravel / cobbles) and was found to be as less erodible than the clay material as defined in (Briaud 2008) within the range of tested velocities.

The erosion rate curve allowed confidence to be gained in the low erodibility of the predominant insitu material, and was used to define an expected profile at the end of the design life of the facilities. It justified the option of leaving the slopes unprotected since the expected erosion due to propeller wash was assessed sufficiently low to not jeopardize the stability of the adjacent facilities during their design life. In addition, the defined erosion rate curve provided the basis for a monitoring and mitigation program, based on comparison of the expected erosion profile against actual bathymetric surveys.

Finally, it shall be noted that the curve has been determined based on a calculated velocity field determined by the Dutch approach and, therefore, the resulting erosion rate curve shall be applied only to velocities obtained using the same approach. This consideration is important when extrapolating the results to other vessel types than the test vessel used in this study.

\section{ACKNOWLEDGMENTS}

The authors would like to acknowledge the support of their respective management teams, to allow them the opportunity to perform the tests. In particular, Rob Scharnell of Chevron, Bert Arien and Sven De Beenhouwer of Dredging International and Kayhan Kamali of Bechtel. Also several colleagues provided valuable insight to the project: among others, Giulia Sforzi, Eric Mary, Walid Harchay, Wannes Van Benneden and Johan Barbaix.

\section{REFERENCES}

Albertson, M. L., Y. Dai, R. Jensen, \& H. Rouse (1950). Diffusion of submerged jets. Transactions of the American Society of Civil Engineers 115(1), 639-664.

BAUW (2005). Principles for the Design of Bank and Bottom Protection for Inland Waterways (GBB) (Mitteilungen 88 ed.). Federal Waterways Engineering and Research Institute, Karlsruhe.

Briaud, J.-L. (2008). Case histories in soil and rock erosion: woodrow wilson bridge, brazos river meander, normandy cliffs, and new orleans levees. Journal of Geotechnical and Geoenvironmental Engineering 134(10), 1425-1447.

PIANC (2015). Guidelines for protecting berthing structures from scour caused by ships. World Association for Waterborne Transport Infrastructure, Brussels.

Verheij, H. (1983). The stability of bottom and banks subjected to the velocities in the propeller jet behind ships, 8th int. In Harbour Congress, Antwerp, Volume 303, pp. 1-11. 